\title{
Mediación virtual en la enseñanza y la instrucción: avances y retos*
}

| Fecha de recibido: 16 de enero del 2020 | Fecha de aprobación: 29 de abril del 2020 |

\author{
María del Pilar García-Chitiva \\ Magíster en Educación \\ Docente e Investigadora en Centro \\ de Estudios Aeronáuticos (CEA) \\ Grupo de Investigación: GINA \\ Rol de investigador: intelectual, \\ experimental, comunicativo \\ https://orcid.org/0000-0001-6776-3422 \\ $\square$ pilargarciach@gmail.com
}

Este artículo de investigación se desarrolla en el marco del proyecto Desarrollo de un modelo de instrucción mediado por TIC, para la instrucción, entrenamiento y evaluación en el puesto de trabajo para controladores de tránsito aéreo. Financiado por el Centro de Estudios Aeronáuticos CEA. 


\section{Mediación virtual en la \\ enseñanza y la instrucción: avances y retos}

avancesyretos
Resumen: Los avances tecnológicos han permeado todas las formas de enseñanza y aprendizaje. Por lo tanto, la instrucción y la capacitación también forman parte de los estudios en los que se documenta el uso de los Entornos Virtuales de Aprendizaje (EVA) para determinar el alcance de las tecnologías virtuales en todos los niveles de enseñanza (desde el aprendizaje de un contenido de una materia en la educación superior hasta la construcción de un barco en la capacitación técnica y laboral). Este artículo tiene dos propósitos. El primero es analizar el estado de la investigación sobre procesos de instrucción mediados por herramientas virtuales. Para ello, se realiza un análisis bibliométrico de las publicaciones realizadas entre 1997 y el 2018, indexadas en la base de datos scopus. El segundo propósito es examinar los principales avances presentados en la literatura científica revisada, para así determinar los desafíos que enfrentan estudios posteriores, principalmente en lo que respecta a procesos de Instrucción, Entrenamiento y Evaluación en el Puesto de Trabajo (IEEPT). Dentro de los hallazgos de esta revisión, se estableció que el incipiente proceso de investigación de la instrucción mediada por tecnología explica la falta de publicaciones indexadas sobre el tema en bases de datos internacionales. También se estableció que, mediante el empleo de las herramientas disponibles para los protocolos de instrucción, como simuladores y software, se pueden adelantar procesos de investigación similares a los documentados en este artículo.

Palabras clave: Aprendizaje virtual; entrenamiento virtual; instrucción virtual.

Abstract: Technological advances have spanned teaching and learning in all its forms. Therefore, instruction and training are also part of studies documenting the use of Virtual Learning Environments (VLEs) to determine the scope of virtual technologies in learning at all levels, from learning a subject topic in higher education to building a boat in technical and work education. This article has two purposes. First, to study the state of research on virtuality-mediated instructional processes through a bibliometric analysis of publications between 1997 and 2018 indexed in the scopus $\odot$ database. Second, to examine the main advances presented in peer-reviewed scientific literature and define the challenges for subsequent studies, mainly for instruction, training, and evaluation in the workplace. According to the results of this study, the first steps about technology-mediated instruction research in the traffic air area have just emerged during recent times as a consequence of the lack of indexed publications on the subject in international databases. Besides, it was established that research processes similar to the studies documented in this article could be carried out by using the tools available for instructional processes (simulators and software).

Keywords: Virtual Learning; Virtual Training; Virtual Instruction.

Resumo: Os avanços tecnológicos têm permeado todas as formas de ensino e aprendizagem. Portanto, a instrução e o treinamento também fazem parte dos estudos que documentam o uso de Ambientes Virtuais de Aprendizagem (AVA) para determinar o alcance das tecnologias virtuais em todos os níveis de aprendizagem (desde a aprendizagem de um conteúdo de uma disciplina no ensino superior até a construção de um navio na capacitação técnica e de trabalho). Este artigo tem dois propósitos. O primeiro é analisar o estado da pesquisa sobre processos instrucionais mediados por ferramentas virtuais. Para isso, é realizada uma análise bibliométrica das que foram realizadas entre 1997 e 2018, indexadas na base de dados scopus. O segundo propósito é examinar os principais avanços apresentados na literatura científica revisada, a fim de determinar os desafios enfrentados pelos estudos subsequentes, principalmente no que se refere aos processos de Instrução, Treinamento e Avaliação no Local de Trabalho. Entre os achados desta revisão, foi estabelecido que o incipiente processo de pesquisa da instrução mediada por tecnologia explica a falta de publicações sobre o assunto indexadas em bancos de dados internacionais. Além disso, foi estabelecido que, usando as ferramentas disponíveis para os processos institucionais, como simuladores e software, processos de pesquisa semelhantes aos documentados neste artigo podem ser realizados.

Palavras-chave: Aprendizagem virtual; Instrução virtual; Treinamento virtual. 


\section{Introducción}

El volumen del tránsito aéreo en Colombia se ha incrementado en los años recientes. Este incremento se debe, entre otras cosas, al crecimiento de la economía que se refleja en las entradas de nacionales y extranjeros al país (Agudelo-Rivera et al., 2019). El aumento en el número de vuelos nacionales e internacionales representa un incremento en las operaciones de tránsito aéreo y, a su vez, demanda una mayor capacidad en el control de este tipo de tránsito. Tal demanda implica el abordaje de dos aspectos fundamentales. El primero es la incorporación de un mayor número de controladores de tránsito aéreo y el segundo, que se establezca un proceso de Instrucción, Entrenamiento y Evaluación en el Puesto de Trabajo (IEEPT). El IEEPT debe tener una fortaleza pedagógica y didáctica que responda a las necesidades del proceso de enseñanza-aprendizaje tanto en términos técnicos y disciplinares (propios de la disciplina o área en la que se aprende) como en términos de las necesidades y preferencias de quien aprende. El presente documento solo se centrará en este segundo aspecto.

El IEEPT se desarrolla bajo las directrices de documentos normativos como el RAC 65 (2019) y el Documento 9868 (OACI, 2016). Según estos, el IEEPT considera el fortalecimiento de las competencias de los controladores de tránsito aéreo en las posiciones tanto de aeródromo como de radar en tiempo real. Este fortalecimiento se debe dar bajo la tutoría de colegas instructores. Los procesos de instrucción de esta naturaleza involucran aspectos técnico-teóricos y prácticos que son abordados en el puesto de trabajo (Tovar, 2015), pero que deben ser igualmente reforzados y trabajados de manera autónoma por quienes participan del proceso. Esto implica que quienes se encuentran en proceso de IEEPT deben realizar repasos o trabajo de estudio y refuerzo autónomo e independiente. Para ello, pueden apoyarse en herramientas tecnológicas que les permitan acceder a los contenidos, evaluaciones y actividades relacionadas con la instrucción que adelantan.
Se ha identificado que desde hace casi tres décadas los procesos de instrucción y enseñanza en todos los niveles han venido incorporando el uso de herramientas o mediaciones tecnológicas, como complemento de los procesos de instrucción presencial (Jardines, 2009). Los procesos de formación técnico, tecnológico y profesional han seguido el camino de la incorporación de tecnologías mediadas por Internet en la enseñanza (García-Chitiva \& Suárez-Guerrero, 2019), lo que ha dado lugar a situaciones de enseñanza-aprendizaje en la modalidad B-learning, que combina la presencialidad con la virtualidad. En este sentido, la evidente y rápida evolución de los adelantos tecnológicos han exigido cambios de paradigma en la comprensión de cómo se enseña y cómo se aprende, y han estimulado las siguientes discusiones: (1) sobre el tipo de mediaciones que pueden ser empleadas para desarrollar procesos de formación a distancia en todos los niveles y procesos de formación (Araiza \& Leal, 2018); (2) sobre el tipo de interacciones que se presentan en dichos procesos, de acuerdo con el tipo de mediación, y el uso que se les da en el proceso educativo o de instrucción. Por lo tanto, la educación a distancia puede comprenderse de dos maneras en función de los tipos de interacciones y mediaciones que se empleen. En la "educación a distancia tradicional" las formas de interacción no están soportadas por lenguajes informáticos (Estévez et al., 2015). En contraste, en la "educación a distancia virtual" se prescinde de los encuentros presenciales y las interacciones entre maestros-estudiantes y estudiantes-estudiantes se soportan exclusivamente por las tecnologías (Rama, 2016).

Según este panorama, las herramientas tecnológicas mediadas por Internet facilitan y potencian los procesos de enseñanza-aprendizaje en todos los niveles y procesos de formación. El presente estudio tiene el propósito de analizar el estado de la investigación sobre procesos de instrucción mediados por la virtualidad (los niveles de publicación sobre el tema, los artículos y fuentes más destacadas, los autores, instituciones de afiliación y países que más trabajan el tópico). También se busca establecer cuáles han sido las tendencias temáticas de trabajo y los retos que este 
tipo de mediación plantea a la instrucción. Particularmente, se quiere establecer cuáles y cuántos de esos trabajos se han desarrollado específicamente sobre IEEPT en el área del control de tránsito aéreo. Con esta última finalidad se pretende establecer el alcance de las investigaciones que hasta la fecha se han desarrollado sobre la inclusión de mediaciones virtuales en esta área. Con esto, se identifica el contexto de antecedentes para el proyecto Desarrollo de un modelo de instrucción mediado por TIC, para la instrucción, entrenamiento y evaluación en el puesto de trabajo para controladores de tránsito aéreo, en el marco del cual se desarrolla el presente estudio bibliométrico. En lo que sigue, se presentan la metodología, los resultados, la discusión y las conclusiones.

\section{Método y materiales}

\section{Tipo de estudio}

El presente estudio es un análisis bibliométrico que analiza las investigaciones sobre mediación virtual en la enseñanza y la instrucción publicadas entre 1997 y el 2018. Un análisis bibliométrico permite describir el estado de investigación sobre un tema a partir del análisis del número de publicaciones y de las citas recibidas por cada publicación (Fernández \& Bueno, 1998; Diem \& Wolter, 2013).

\section{Procedimiento de recolección y análisis de datos}

Uno de los propósitos principales de esta investigación era identificar las investigaciones que hubiesen desarrollado IEEPT mediado por TIC en el control del tránsito aéreo, que se encontraran en la base de datos SCOPUS ${ }^{1}$. Para cumplir este objetivo, se establecieron

1 Se seleccionó la base de datos scopus, ya que esta indexa el contenido de 24600 revistas de todo el mundo en diferentes áreas. Esto permite un mayor alcance en la revisión de una temática de interés global. los términos clave que describieran los temas de interés: "aprendizaje virtual", "entrenamiento", "instrucción" y "tráfico aéreo". Se buscaron los términos en el idioma inglés, ya que la búsqueda en español no generó ningún tipo de resultado. Se emplearon dos estrategias de búsqueda. La primera fue usar las siguientes combinaciones de palabras clave: "air traffic" AND "virtual learning" AND "training" AND "instruction"; "air traffic" AND "virtual learning"; "virtual training" AND "air traffic", y "virtual instruction" AND "air traffic". Como estas combinaciones de búsqueda no dieron ningún resultado, se procedió a eliminar las palabras especificas relacionadas con el tránsito aéreo (air traffic).

La segunda estrategia de búsqueda fue usar la combinación de palabras clave "virtual learning" AND "training" AND "instruction". Con esta búsqueda se obtuvieron 255 documentos, distribuidos así: 4 libros, 13 capítulos de libro, 155 artículos de conferencias, 71 artículos, 2 artículos en prensa, 6 artículos de revisión de conferencias y 4 revisiones. Se consideró que los 255 documentos generados en esta búsqueda conformaban un corpus robusto para realizar un análisis bibliométrico que permitiera describir los detalles de las investigaciones e identificar las tendencias desarrolladas en esta temática de investigación. Por esta razón no se realizaron más búsquedas.

Para el tratamiento de los datos, se realizó un análisis estadístico descriptivo de la muestra y se emplearon técnicas de visualización de datos. Se desarrollaron estadísticas descriptivas, técnicas de visualización de datos en el lenguaje de programación $\mathrm{R}$ (Wickham, 2009) y se calculó el aumento de publicaciones por año mediante el paquete Bibliometrix (Aria et al., 2017). Igualmente, se usaron técnicas de visualización y análisis de redes (Cherven, 2015), desarrolladas en la interfaz Biblioshiny de Bibliometrix, con el fin de identificar los documentos más relevantes, los autores más representativos y la colaboración entre investigadores de diferentes países. Para analizar la tendencia y la evolución temática de las investigaciones sobre el tema, se emplearon técnicas de visualización de redes con la herramienta VosViewer (Perianes-Rodriguez et al. , 2016). 


\section{Resultados}

De los 255 documentos analizados, 38 fueron publicados por un único autor, mientras que 217 fueron escritos en coautoría por al menos tres autores.

Con respecto al comportamiento de la producción de las publicaciones, se estableció que, específicamente sobre el tema, las primeras publicaciones surgen en 1997 (figura 1). También se identificó que en la primera década (desde 1997 hasta el 2008) la producción científica al respecto fue pequeña, ya que las publicaciones solo ocuparon el 17,6 \% del total de los documentos analizados. En contraste, en la siguiente década (desde el 2009 hasta el 2018) se dio un incremento significativo, pues en esta franja de tiempo se publicó el 82,3\% del total de las publicaciones. Estos primeros hallazgos coinciden con la llegada de herramientas tecnológicas soportadas por Internet (las cuales serán comentadas más adelante, en la discusión) y con el incremento de la oferta de programas de instrucción con mediación virtual (ya sea total - programas $100 \%$ virtuales-o parcial -Blended Learning-) en todos los niveles educativos.

Tal como se indica en la figura 2, las áreas del conocimiento en las que se concentra el mayor número de publicaciones sobre enseñanza e instrucción mediada virtualmente son ciencias de la computación, ingeniería, matemáticas y salud (medicina). Estas son áreas en las que se involucran procesos de instrucción que emplean laboratorios virtuales, ambientes

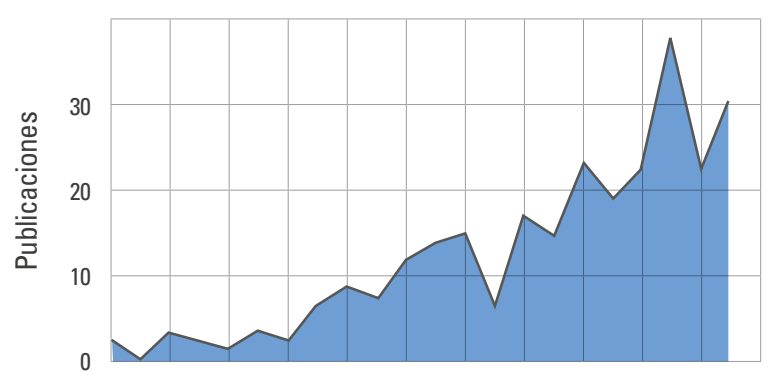

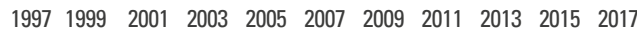
Año

Figura 1. Comportamiento de las publicaciones por año Fuente: elaboración propia. de simulación, ambientes virtuales de realidad aumentada, entre otros.

Con respecto a la investigacion sobre la mediación virtual en la instrucción, en la figura 3 se observa que, aunque la oferta de procesos de instrucción y el crecimiento de la mediación virtual en ellos se documenta desde la década de 1990, solo algunos países desarrollan estudios en colaboración al respecto. Los países identificados dan cuenta de la ubicación de la institución de afiliación de los autores. Se observa que Estados Unidos es el país en el que se da el mayor número de investigaciones (nueve) sobre el tema desarrolladas en colaboración con investigadores de países como Australia, Canadá, China, Francia, Grecia, India, Rumania, Holanda y Taiwán. Así mismo, en el caso de España y el Reino Unido, cada uno tiene siete publiciones realizadas en colaboración con investigadores de países como Dinamarca, Francia, Grecia, Irlanda, Italia, Lituania, Noruega, Australia, Brasil, Ecuador, Alemania y Guatemala. Dentro de las diez instituciones más sobresalientes por el número de documentos publicados, se encuentran dos instituciones británicas, dos españolas, una iraní, una brasilera, una norteamericana, una irlandesa y una koreana. Este grupo de colaboración entre inter-instituciones e inter-países muestra que la investigación en Latinoamérica tiene una reducida presencia en publicaciones indexadas en bases de datos como scopus. No obstante, pueden existir publicaciones en fuentes latinoamericanas pero que no son refrenciadas aquí debido a que el análisis se centra exclusivamente en scopus.

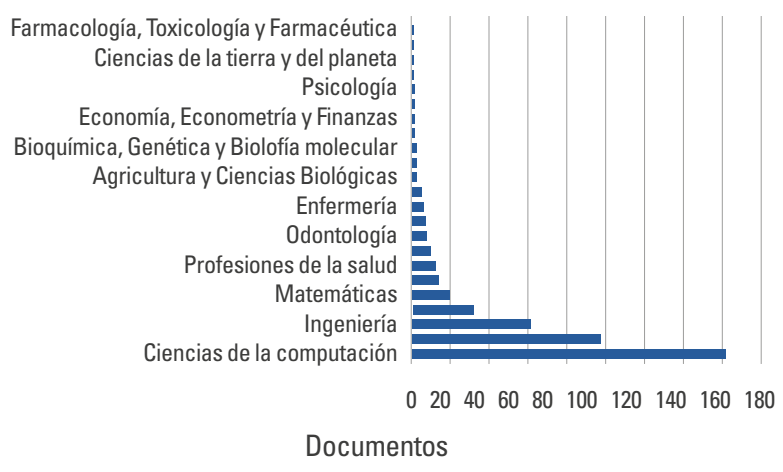

Figura 2. Áreas de publicacion sobre el tema

Fuente: elaboración propia. 


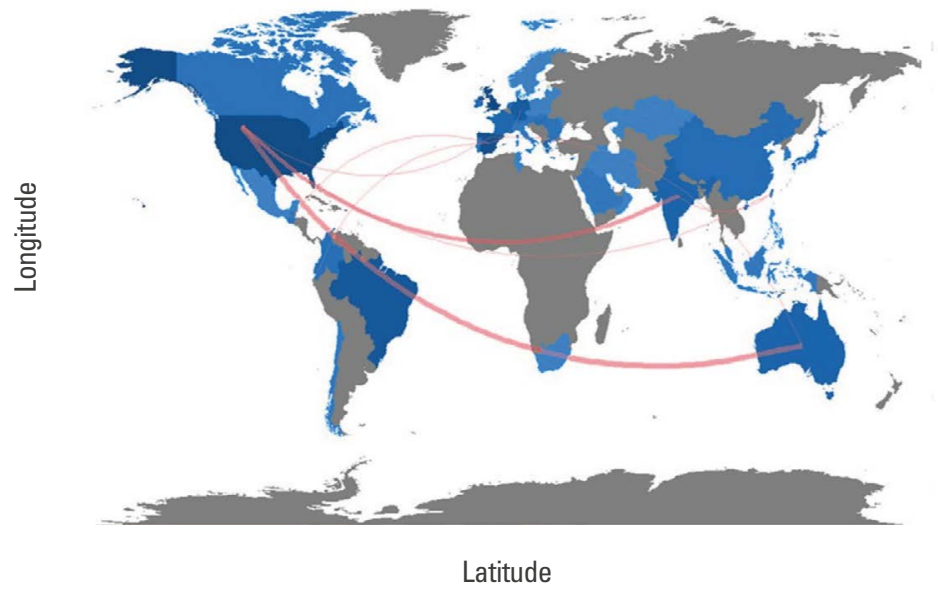

Institución

Artículos

University of Nottingham

5

King's College London Dental Institute

4

Pontificia Universidade Católica do Rio de Janeiro

Shiraz University of Medical Sciences

Universidad Politécnica de Madrid

University of Salamanca

Armstrong Atlantic State University

Charles Sturt University

Dublin City University

Far East University

Figura 3. Mapa de colaboración en la investigación sobre mediación virtual en la enseñanza y la instrucción Fuente: elaboración propia.

Al analizar las publicaciones más destacadas, se encuentra que la mayoría de los veinte documentos más citados (tabla 1) documentan al empleo de laboratorios virtuales, de simulación y al uso de metododologías comparadas con la virtual. Las dos publicaciones más citadas ("Web-Based Virtual Learning Environments: A Research Framework and a Preliminary Assessment of Effectiveness in Basic IT Skills Training" $y$
"Learning in a Virtual World: Experience With Using Second Life for Medical Education") analizan la utilidad de entornos de realidad aumentada para la enseñanza y el entrenamiento. En la tabla 1 también se muestra el cuartil al que pertenecen las fuentes en las que se publicaron dichos documentos, según Scimago JR, y su índice de impacto. Estos datos permiten visibilizar la notoriedad de estos documentos para el campo de estudio.

Tabla 1

Listado de las 20 publicaciones más citadas

\begin{tabular}{|c|c|c|c|c|}
\hline (Piccoli et al., 2001) & $\begin{array}{l}\text { Web-Based Virtual Learning Environments: A Research Framework and a } \\
\text { Preliminary Assessment of Effectiveness in Basic IT Skills Training }\end{array}$ & 726 & 03 & 25 \\
\hline (Wiecha et al., 2010) & $\begin{array}{l}\text { Learning in a Virtual World: Experience With Using Second Life for } \\
\text { Medical Education }\end{array}$ & 132 & 01 & 54 \\
\hline (Anohina, 2005) & Analysis of the Terminology Used in the Field of Virtual Learning & 51 & NA & 7 \\
\hline (Larvin, 2009) & E-Learning in Surgical Education and Training & 44 & 01 & 91 \\
\hline (Green et al., 2006) & $\begin{array}{l}\text { The Development and Evaluation of the Use of a Virtual Learning Environment } \\
\text { (Blackboard 5) to Support the Learning of Pre-Qualifying Nursing Students } \\
\text { Undertaking a Human Anatomy and Physiology Module }\end{array}$ & 37 & 02 & 34 \\
\hline (Limniou et al., 2009) & $\begin{array}{l}\text { Integration of Simulation Into Pre-Laboratory Chemical Course: Computer } \\
\text { Cluster Versus Webct }\end{array}$ & 32 & 01 & 50 \\
\hline (Stonebraker \& Hazeltine, 2004) & Virtual Learning Effectiveness: An Examination of the Process & 32 & 01 & 131 \\
\hline (Corrigan et al., 2008) & $\begin{array}{l}\text { "SURGENT"-Student e-Learning for Reality: The Application of Interactive } \\
\text { Visual Images to Problem-Based Learning in Undergraduate Surgery }\end{array}$ & 27 & 01 & 249 \\
\hline (Clarke et al., 2005) & A Strategic Approach to Developing e-Learning Capability for Healthcare & 27 & 01 & 189 \\
\hline
\end{tabular}




\begin{tabular}{|c|c|c|c|c|}
\hline (Kim \& Schniederjans, 2004) & The Role of Personality in Web-based Distance Education Courses & 26 & Q1 & 45 \\
\hline (Kleinpell et al., 2011) & Web-Based Resources for Critical Care Education & 25 & 02 & 34 \\
\hline (Maxwell, 2012) & $\begin{array}{l}\text { An Agenda for UK Clinical Pharmacology: How Should Teaching of } \\
\text { Undergraduates in Clinical Pharmacology and Therapeutics Be Delivered } \\
\text { and Assessed? }\end{array}$ & 23 & Q1 & 149 \\
\hline (Yoshida et al., 2011) & $\begin{array}{l}\text { Development of a Multi-Layered Virtual Tooth model for the Haptic Dental } \\
\text { Training System }\end{array}$ & 23 & 02 & 50 \\
\hline (Raynor \& Iggulden, 2008) & $\begin{array}{l}\text { Online Anatomy and Physiology: Piloting the Use of an Anatomy and } \\
\text { Physiology e-Book-VLE Hybrid in Pre-Registration and Post-Qualifying } \\
\text { Nursing Programs at the University of Salford }\end{array}$ & 22 & 01 & 65 \\
\hline (Raupach et al., 2009) & Web-Based Collaborative Training of Clinical Reasoning: A Randomized Trial & 21 & 03 & 69 \\
\hline (Lytvyn et al., 2016) & $\begin{array}{l}\text { A Method for Constructing Recruitment Rules Based on the Analysis of a } \\
\text { Specialist's Competences }\end{array}$ & 19 & 01 & 73 \\
\hline (Moazami et al., 2014) & $\begin{array}{l}\text { Comparing Two Methods of Education (Virtual Versus Traditional) on Learning } \\
\text { of Iranian Dental Students: A Post-Test Only Design Study }\end{array}$ & 19 & 01 & 116 \\
\hline (Shakhovska et al., 2017) & $\begin{array}{l}\text { Intelligent Systems Design of Distance Learning Realization for Modern Youth } \\
\text { Promotion and Involvement in Independent Scientific Researches }\end{array}$ & 18 & 01 & 195 \\
\hline (Pinto-Llorente et al., 2017) & $\begin{array}{l}\text { Students' Perceptions and Attitudes Towards Asynchronous Technological } \\
\text { Tools in Blended-Learning Training to Improve Grammatical Competence in } \\
\text { English as a Second Language }\end{array}$ & 17 & 03 & 25 \\
\hline (San Diego et al., 2012) & $\begin{array}{l}\text { Researching Haptics in Higher Education: The Complexity of Developing } \\
\text { Haptics Virtual Learning Systems and Evaluating Its Impact on } \\
\text { Students' Learning }\end{array}$ & 17 & 01 & 54 \\
\hline
\end{tabular}

Fuente: elaboración propia.

Mediante análisis de las palabras clave, se identificó que son 645 los términos clave más comúnmente empleados por los autores en los 255 documentos analizados. Esto permitió identificar dos aspectos fundamentales que describen la tendencia sobre los objetos de estudio.

El primer aspecto es la tendencia temática, que varía por épocas. En la figura 4 se muestran nodos de colores (azul oscuro, verde oscuro, verde claro y amarillo). Se observa que, dados los términos agrupados en los nodos de color azul oscuro "instrucción médica", "aprendizaje a distancia", "ambientes virtuales", "aprendizaje a lo largo de la vida", "Entornos Virtuales de Aprendizaje" -VLE, por sus siglas en inglés-, "instrucción basada en Internet "y "diseño instruccional"), la investigación antes del 2010 se enfocó en perfilar los entornos virtuales de aprendizaje (EVA). A partir del 2000 se empezaron a modificar y sofisticar las primeras versiones de estos entornos, que tuvieron su origen en la década de 1990. Con respecto a la educación en áreas como la medicina y la ingeniería, se adelantaron estudios en los que se instruía a distancia con la mediación tecnológica y se avanzó en la creación de modelos de diseño instruccional. Lo mismo sucedió en áreas como la física o las ciencias sociales, entre otras.

Por su parte, los nodos de color verde oscuro abarcan las publicaciones identificadas con las palabras clave "e-learning", "educación", "evaluación y entrenamiento mediado por Internet" (ICT, por sus siglas en inglés). Esta segunda tendencia, dada entre el 2011 y el 2012, muestra que los estudios dieron un giro hacia la comprensión de cómo enseñar y evaluar en los escenarios mediados por Internet. Es decir, antes del 2011 se adecuaron los entornos y entre el 2011 y el 2012 se empezaron a interpretar y definir las maneras de enseñar, aprender en ellos y evaluar. 
Los nodos de color verde claro (que encapsulan las palabras clave "entrenamiento y enseñanza", "aprendizaje en línea", "educación superior", "aprendizaje combinado", "educación asistida por computador", "simulación" - verde claro ligeramente más oscuro-, "aulas virtuales", "tecnologías e información", "Second Life", "realidad virtual", "diseño curricular", "accesibilidad y entornos virtuales" -verde ligeramente más claro-) muestran que entre el 2013 y el 2016 hay una tercera tendencia, derivada de las anteriores. En este periodo se busca determinar el efecto de las adecuaciones en los entornos virtuales creados anteriormente (como en el caso de Moodle) y de la creación de nuevos entornos virtuales (que incorporaron tecnologías de realidad virtual, como en el caso de Second Life) sobre las interacciones y los procesos de enseñanza-aprendizaje.

Finalmente, los nodos amarillos (que representan las palabras clave "entornos virtuales de aprendizaje”, "gamificación”, "información y comunicaciones" y "comunidades virtuales") muestran que el objeto de estudio predilecto entre el 2016 y el 2018 fue las implicaciones del uso de herramientas de información y comunicación (entre docentes-estudiantes y estudiantes-estudiantes) en los entornos virtuales. Igualmente, en ese periodo se analizó el impacto de estas herramientas para el desarrollo de trabajo colaborativo y la consolidación de comunidades de aprendizaje. También se refleja aquí un interés por analizar las implicaciones del juego en los procesos de enseñanzaaprendizaje virtual.

El segundo aspecto relevante que se analiza a través de la red de coocurrencia en la figura 4 es el agrupamiento palabras clave (grupos o clusters). Los clusters pueden entenderse como microconjuntos de elementos que están altamente conectados entre sí y que hacen parte de un conjunto de elementos más robusto (Cherven, 2015). Los clusters se conectan por segmentos de acuerdo a su nivel de proximidad; en este caso, de acuerdo a su nivel de coocurrencia. Se observan algunos nodos (palabras clave) más prominentes que otros; por ejemplo, e-learning. Este término en particular, perteneciente al cluster 3 , se conecta con al menos cinco de los seis nodos. Evidentemente, esto se debe a la popularización de la educación mediada por Internet. En el primer cluster se ubican las palabras clave "blended learning", "computer assisted instruction", "higher education", "ICT", "medical education", "Moodle", "teacher training" $y$ "virtual learning environment". Los estudios que emplean estos términos clave analizan el entrenamiento en áreas diversas empleando los EVA y, en algunos casos, simulaciones. Tanto los Eva como las simulaciones son mediados por los entornos virtuales. Por ejemplo, Halpin et al. (2015) desarrollaron un módulo de capacitación en seguridad eléctrica, basado en simulación, construido en una plataforma que empleó el software Blender y Unity3. El objetivo de este módulo era proveer a los empleados de empresas de diferentes áreas en un proceso de capacitación virtual. El diseño del módulo contempló las áreas de aprendizaje del nivel superior propuestas en la taxonomía de Bloom (aplicación, análisis, síntesis y evaluación).

En otro estudio, Pinto-Llorente et al. (2017) analizaron la percepción de 358 estudiantes universitarios de un programa de segunda lengua sobre la efectividad de herramientas asincrónicas, en una modalidad blended learning, para cumplir con los objetivos de la materia Morfosintáxis. Los resultados indicaron que los estudiantes percibieron mejores logros en las evaluaciones y en el aprendizaje de los contenidos. Igualmente, los estudiantes resaltaron la relevancia de esta metodología para la autonomía y la autogestión en el aprendizaje, ya que el complemento educativo que proporciona la virtualidad les permitió seguir sus propios ritmos de aprendizaje.

Dentro de las publicaciones relacionadas en el primer cluster se encuentran aquellas que analizan diversas metodologías para potenciar el aprendizaje en los entornos virtuales. Por ejemplo, Elledge et al. (2018) analizaron el efecto de la metodología de aula invertida, en un curso de medicina oral, sobre el logro y percepción de confianza en lo que se aprende. Se compararon los resultados de un grupo experimental (expuesto a trabajo virtual en contenidos de la materia) con los de un grupo control (que recibió orientación mediante la metodología tradicional de trabajo en aula, expositiva, a cargo de docentes y expertos). Los resultados mostraron que, si bien no había 
diferencias en el logro, sí hubo diferencias en la percepción de confianza en lo que se aprende.

Otros estudios se enfocaron en identificar el potencial de diversos EVA para el aprendizaje dentro de la metodología virtual y en determinar cómo la capacitación de docentes y actores educativos es fundamental para el aprovechamiento efectivo de la enseñanza-aprendizaje virtual. Por ejemplo, Kavitha \& Lohani (2018) revisaron algunos LMS abiertos de uso masivo (como Moodle, Ilias, Opigno, entre otros) con el fin de identificar las características de los LMS, las herramientas de inteligencia artificial que en estos se emplean y establecer un modelo de uso pedagógico de los LMS que facilite el aprendizaje de los estudiantes, independientemente de sus preferencias para aprender (estilos de aprendizaje). Con respecto a la necesidad de capacitar a docentes y actores educativos,
Penafiel et al. (2018) desarrollaron un programa de capacitación en plataformas para la creación de espacios virtuales de aprendizaje, dirigido a los educadores de ingeniería, con el fin de incrementar sus competencias en nuevas tecnologías y en las metodologías de diseño de enseñanza aplicadas al diseño de entornos de aprendizaje en línea. Con esto, se buscaba fortalecer los procesos de enseñanza-aprendizaje de los futuros ingenieros. Dicha capacitación consideró fundamentos pedagógicos, estrategias de instrucción y aprendizaje, así como manejo de sistemas de gestión de la información. Los resultados de la capacitación mostraron que los educadores participantes incrementaron su conocimiento y manejo de los sistemas LMS, así como sus competencias para proponer y desarrollar actividades en línea con los estudiantes y acompañar el proceso de formación en esta modalidad.

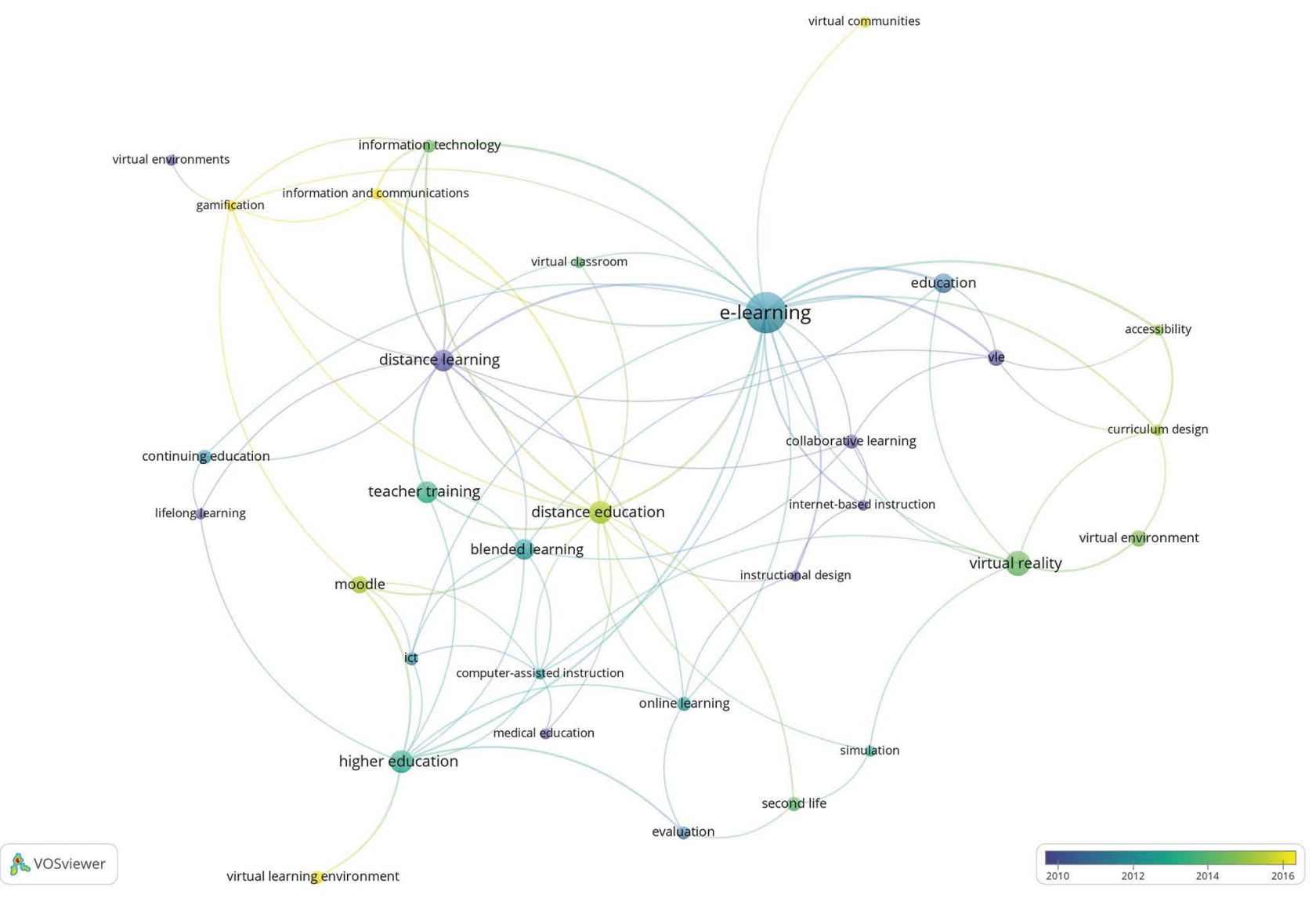

Figura 4. Red de coocurrencia de palabras clave

Fuente: elaboración propia

Revista Ciencia y Poder Aéreo | vol. 15 n. 1 | enero-junio del 2020 | pp. 161-177 | ISSN 1909-7050 E-ISSN 2389-9468 | https://doi.org/10.18667/cienciaypoderaereo.645 
El segundo cluster agrupa las palabras clave "accessibilty", "curriculum design", "education”, "virtual environment", "virtual reality" y "VLE". Los estudios en los que aparecen estos términos analizan diseños curriculares y propuestas educativas virtuales que brinden posibilidades de acceso a todas las personas, independientemente de su condición, y que brinden recursos a los docentes e instructores para saber orientar los procesos académicos de los aprendices o estudiantes. En esta línea, Amado-Salvatierra, et al. (2014) propusieron una estrategia de capacitación para mejorar los índices de accesibilidad en los EVA. La estrategia fue usada en siete países latinoamericanos, en el marco de un workshop. La propuesta de capacitación estuvo dirigida a desarrolladores, adecuadores y personal vinculado en la gestión de EVA educativos, en ella se definieron seis aspectos inherentes al uso de EVA con el fin de establecer las características de acceso óptimas que los Eva debían tener. Se resalta que un Entorno Virtual de Aprendizaje debe incluir: lectura; análisis y estudio de contenido educativo; una autoevaluación del conocimiento por cada unidad de aprendizaje; un test de evaluación del conocimiento teórico; participación en foros de discusión que promueven la socialización y las intervenciones; el estudio a través de estudios de caso resueltos, y la exploración de contenido multimedia. Los autores concluyeron que esta experiencia se debe difundir ampliamente en toda Latinoamérica para fortalecer y garantizar el acceso a EVA que permitan la igualdad de oportunidades en la población en general, y especialmente en la población diversa.

Giannakou, et al. (2016) realizaron un estudio de caso con respecto a una capacitación que garantice el acceso y el desempeño favorable de los aprendices o estudiantes en los procesos de instrucción o formación en los EVA. La capacitación estaba dirigida a instructores y docentes. Este estudio analizó el uso de una capacitación sobre inteligencia emocional dirigida a un grupo de doce educadores de un programa de ingeniería de sistemas. La capacitación se desarrolló en el entorno Second Life y su propósito era brindar herramientas pedagógicas sobre Aprendizaje Social y Emocional (SEL, por sus siglas en inglés) y fortalecer el uso de estas herramientas. Los resultados mostraron que trabajar en este entorno permitió a los docentes adoptar y adecuar estrategias para apoyar a los estudiantes en la resolución de problemas, procesos de regulación, generación de actividades que promocionen la autorregulación, entre otras.

Otro estudio asociado a este cluster investigó el efecto de la incorporación de un EVA en un proceso de capacitación e instrucción en construcción de navíos (Souppez, 2015). Este fue un estudio de caso en el que, durante un periodo de ocho meses, se analizó el desempeño de un grupo de estudiantes de un curso de construcción de barcos en una universidad del Reino Unido. En el estudio se optimizaron los recursos de un EvA para reforzar la instrucción en la construcción de los navíos y facilitar la comunicación entre los constructores y los clientes. Los resultados mostraron que el empleo y la optimización de los Eva permitieron a los aprendices avanzar de manera autónoma en las competencias que requieren para realizar la construcción. Igualmente, se mostró que el empleo de las herramientas de comunicación face to face facilita e incrementa el nivel de precisión en la elaboración de los barcos según lo que el cliente requiere.

En el tercer cluster (que agrupa las palabras clave "collaborative learning", "e-learning", "instructional design" e "Internet base instruction") se asocian los estudios e investigaciones que analizan los diseños de instrucción y de EVA para resaltar las características que resultan efectivas para la enseñanza y el aprendizaje. En este marco, Hernández-Leo et al. (2013) hicieron un análisis de las opiniones de los usuarios de tres instituciones educativas (en capacitación vocacional, educación superior y educación de adultos virtual) con respecto a los elementos que deben considerarse en un Diseño de Entornos de Aprendizaje Integrado (ILDE, Integrated Learning Design Environment). Se les preguntó a las personas sobre aspectos que deben o no ser considerados en un ILDE, aspectos tales como selección de una herramienta de diseño de aprendizaje; poder producir o coproducir un diseño de aprendizaje; poder compartir un diseño de aprendizaje; poder desarrollar e implementar un diseño de aprendizaje en EVA; poder proporcionar comentarios y reflexiones, y poder explorar diseños, instancias y comentarios. 
A partir de las respuestas de cuestionarios, entrevistas en línea y trabajo grupal, los investigadores identificaron que un ILDE debe permitir a los maestros seleccionar herramientas para crear, producir, elaborar, compartir, evaluar e implementar diseños de aprendizaje en los entornos virtuales.

En la misma línea, Gutiérrez-Esteban et al. (2016) evaluaron los diseños y metodologías de enseñanza utilizados en un curso del plan de formación sobre EVA. Este plan de formación se realizó con cuarenta profesores de diferentes áreas de la Universidad de Extremadura, España. A través de grupos focales, los investigadores identificaron los beneficios y aspectos por mejorar en el uso de las Aulas Virtuales Síncronas (svc, Synchronous Virtual Classrooms). Los resultados reflejaron que los escenarios virtuales son los más adecuados para capacitar a los docentes que se desempeñan en esta modalidad, pues es en esos escenarios en lo que se fortalecen de manera directa y auténtica las competencias digitales. Por otro lado, se estableció que este tipo capacitaciones fortalece las habilidades tecnológicas, informativas y pedagógicas de los docentes. Estas habilidades permiten que quien enseña o instruye ayude a los estudiantes a tener ideas colaborativas, a construir conocimiento compartido, y a intercambiar, debatir y reflexionar conjuntamente. Estas son actividades clave en este entorno.

En un estudio más reciente, Daineko et al. (2018) desarrollaron un laboratorio virtual de física (con visualización 3D) en la Universidad Internacional de Tecnologías de la Información de Kazajstán. El laboratorio virtual se desarrolló para el programa de Ingeniería Informática y Telecomunicaciones, y estaba destinado al uso de los estudiantes de estas carreras. El esquema de trabajo que guio el desarrollo de este laboratorio virtual contiene unas pautas que orientan al estudiante para plantear y desarrollar un objetivo de trabajo, acceder a material teórico, usar un dispositivo experimental y adelantar un orden de ejecución de la obra. Los primeros resultados de la implementación del laboratorio indicaron que, dada su naturaleza virtual, este puede ser empleado por estudiantes de otras universidades, regiones y carreras afines a las ciencias naturales y programas técnicos.
En el marco del cuarto cluster se agruparon los términos clave "distance education", "evaluation", "online learning", "Second Life" y "simulation". Estos términos se vinculan con estudios que analizaron la efectividad en el uso de entornos de realidad virtual para el aprendizaje, evaluaron y analizaron las percepciones sobre el aprendizaje bajo esta modalidad e idearon programas para favorecer el aprendizaje remoto (virtual) en áreas diversas, entre ellas el área de la salud. Flores et al. (2012) desarrollaron un sistema de aprendizaje multiagente (en el que el agente de dominio eran las actividades y contenidos dispuestos en el EVA; el agente de aprendizaje eran los profesionales que tomaron el curso y el agente de mediación era el profesional que hizo las veces de tutor u orientador) para profesionales de la salud. El sistema era una Simulación para la toma de decisiones en el Servicio de Atención Médica (SimDeCS, Simulation for Decision Making in the Health Care Service). El sistema estaba diseñado para realizar simulaciones de casos clínicos complejos incluidos en un programa de educación, capacitación y calificación virtual dirigido a profesionales de la salud en Brasil. Mediante este sistema se esperaba mejorar las competencias de los profesionales de esta área en la atención mediante la práctica técnica de casos clínicos complejos.

Con respecto al tema de la evaluación en los EVA, Cuber et al. (2014) desarrollaron un programa de capacitación basado en un entorno para el entrenamiento de habilidades de evaluación (pensamiento analítico y crítico, resolución de problemas, sentido de la ética, toma de decisiones y trabajo en equipo). Los estudiantes de último año de formación profesional tomaron la capacitación. El entorno, denominado EDECOM-DevalSimWeb, permitía a los estudiantes participar activamente en procesos de evaluación en línea mediante el uso del entorno Evalсомıх web. Los estudiantes también podían participar en foros en el entorno de trabajo, en wikis y juegos de simulación y, en general, en cualquier actividad dispuesta para el fin formativo. Los investigadores establecieron que el entrenamiento en habilidades de evaluación puede ser desarrollado con éxito en un entorno virtual y que dichas habilidades 
pueden ser potenciadas más ampliamente si se complejizan las tareas a desarrollar.

Más recientemente, en relación con la percepción de estudiantes y docentes sobre el aprendizaje en los EVA, Abutakka, Maciel et al. (2018) examinaron la experiencia de implementación de educación a distancia en cursos de Ciudadanía y Control Social mediados por Internet. El examen se realizó a través de un análisis descriptivo de las respuestas obtenidas por los estudiantes que tomaron el curso. Se les solicitó que dieran su opinión sobre los aspectos a mejorar en los componentes pedagógicos y técnicos, así como ajustes que realizarían al curso de educación a distancia, Ciudadanía y Control Social. Los resultados indicaron que los participantes valoraron como pertinente el desarrollo procesos de formación que propician la participación y la educación en ciudadanía y democracia a distancia en los EVA. Estos procesos, en opinión de los participantes, permiten que el acceso y la participación sean posibles para todos, y que este tipo de formación esté al alcance de todos, independientemente del lugar geográfico y tiempo (uso horario) en el que se encuentren.

En el quinto cluster se agruparon las palabras clave "continuing education", "distance learning", "lifelong learning" y "virtual classroom". Los estudios relacionados con dichos términos analizaron las características de la enseñanza en los EVA y, a partir de estudios de metodología comparada, identificaron la eficacia del aprendizaje a través de los EVA. El estudio comparativo adelantado por López et al. (2014) se centró en el desempeño de 1133 estudiantes en un programa vocacional de gestión administrativa durante 11 años. Las comparaciones se realizaron de acuerdo con las variables definidas inicialmente en el estudio: modalidad virtual o modalidad presencial; horarios de las clases, mañana-media tarde, media tarde-iniciada la noche, o distancia virtual; grupo, género, edad y año de terminación del programa. También se tuvieron en cuenta las siguientes variables después de haber obtenido y organizado los datos: calificación promedio recibido en las clases durante el proceso del curso vocacional; número de asignaturas aprobadas; número de sujetos reprobados; número de asignaturas en las que el alumno se retiró formalmente de la asignatura antes de la hora del examen; número de asignaturas en las que el alumno no asistió a los exámenes; nivel de avance de los estudiantes (indicado por el paso o no al siguiente nivel en el curso), y permanencia o retiro. Los resultados mostraron que existieron diferencias en los rangos de edad entre los estudiantes de la mañana, la tarde-noche y la modalidad virtual-distancia. Los estudiantes más jóvenes (18 años) tomaban las clases de la mañana. En la tarde-noche, la edad fue en promedio de 21 años. Finalmente, en la modalidad virtual-distancia, se encontraron estudiantes de 25 años en adelante. Se identificó que el programa cubrió las necesidades educativas de los estudiantes a partir de una oferta flexible y variada para el acceso. Con respecto a la deserción y el logro, los investigadores encontraron que los estudiantes de la modalidad virtual desertaron más y que el desempeño fue comparable en los tres grupos de estudiantes (diurnos, tarde-noche y virtual-distancia).

Silva \& López (2015) realizaron un experimento comparativo con grupo control. En el estudio se contrastó el logro de dos grupos, uno compuesto por cuarenta y cuatro personas (grupo control) y otro compuesto por cincuenta y tres (grupo experimental). Las participantes fueron enfermeras expuestas a procesos de capacitación de idéntico contenido. El grupo experimental recibió la capacitación en un EVA y el grupo control lo hizo presencialmente. Aunque los resultados evidenciaron que el desempeño de los dos grupos fue estadísticamente similar, la valoración de las pruebas de conocimiento fue mejor en el grupo experimental, en contraste con el grupo control. Esto evidenció que la educación a distancia mediada por Internet puede ser una alternativa efectiva para la formación de profesionales en esta área, principalmente con respecto a la capacitación en conocimientos con altos niveles de complejidad.

En un estudio longitudinal, Bogossian et al. (2015) analizaron la calidad de los cursos de educación a distancia virtual desarrollados en una universidad de Brasil en un periodo de siete años. Durante este periodo, 5892 estudiantes evaluaron a 9000 docentes mediante una encuesta sobre la calidad educativa. La encuesta 
se refería a distintos temas: materiales didácticos, mediación pedagógica, entorno de aprendizaje virtual, metodología, apoyo académico y técnico del alumno. Los resultados mostraron que, en general, los estudiantes consideran que la educación a distancia virtual es de calidad cuando existe una efectiva y continua mediación pedagógica de parte del maestro y cuando el diseño de enseñanza-aprendizaje fomenta la colaboración.

Finalmente, el sexto cluster agrupó las palabras clave "gamification", "information and communication", "information technology" y "virtual environments". Los estudios relacionados con este cluster analizaron los niveles de inmersividad; el efecto de los entornos que emplean realidad virtual sobre el aprendizaje; la relevancia de formar a docentes y estudiantes en habilidades tecnológicas para un desempeño efectivo en los EVA, y la influencia de la gamificación en el logro y el aprendizaje. Al respecto, Gordon \& Brayshaw (2017) examinaron los elementos que deben ser considerados en el desarrollo de procesos de gamificación efectivos para el aprendizaje en educación superior virtual. Para ello, examinaron las condiciones óptimas que debe tener un entorno virtual que favorezca el aprendizaje a través de la gamificación. Entre estas condiciones se destacan el sentido de presencia (que el entorno proporcione interacciones creíbles), las modalidades (posibilidad de diferentes formas de comunicarse - chat, llamadas, video llamadas-), la inclusividad (considerar en el diseño las posibilidades de acceso para personas con discapacidad), la evaluación (los aspectos que midan el compromiso, como la presencia puntual del estudiante, y que los estudiantes mismos puedan evaluar la efectividad de la gamificación en su aprendizaje) y la motivación y compromiso (el diseño del juego, las características educativas que permitan al estudiante aprender y comprometerse con el material).

Por otra parte, Häfner et al. (2013) examinaron el efecto del nivel de inmersividad en el nivel de conocimiento adquirido durante una tarea de memorización. Se esperaba establecer de este modo si los diseños de los entornos y las interacciones que estos propician inciden sobre el aprendizaje. Los resultados mostraron que ni los tipos de movimiento ni los niveles de inmersión en entornos virtuales afectan el rendimiento de la memorización de manera significativa.

Desde otro enfoque, centrado en los recursos o insumos que deben tener el docente y el estudiante en la modalidad virtual, Letouze et al. (2017) desarrollaron una propuesta de enseñanza de gestión de tecnología para procesos de formación en los EVA, dirigida a docentes y estudiantes. Para ello, revisaron los artículos científicos relacionados con la enseñanza o educación en gestión de tecnología en los EVA disponibles en las bases de datos ACM, Emerald, Eric, IEEE Xplore Digital Library, ISI Web of Science, scielo, ScienceDirect, Springer, Wiley, y Google Scholar. Este análisis les permitió establecer que es preciso desarrollar procesos de enseñanza interdisciplinares en la enseñanza virtual, particularmente, en gestión de las tecnologías usadas en los EVA dirigidos a estudiantes y docentes para que el proceso de enseñanza-aprendizaje sea efectivo. También señalaron que dicha enseñanza debe ser abordada en el contexto propio de la disciplina, del área de formación, y que debe servirse de situaciones y problemáticas en las que la mediación (las tecnologías) permita llegar a la meta de aprendizaje para que el estudiante adquiera también habilidades tecnológicas.

\section{Discusión y conclusiones}

El objetivo de este trabajo era analizar el estado de la investigación sobre procesos de instrucción mediados por la virtualidad a partir del volumen de publicación, los datos bibliométricos y las temáticas abordadas. Con estos datos se podría establecer cuáles y cuántas de esas publicaciones fueron desarrolladas en el marco de la Instrucción, Entrenamiento y Evaluación en el Puesto de Trabajo (IEEPT) en el área del control de tránsito aéreo. Se pudo determinar que, a la fecha, no hay evidencia de investigaciones indexadas en la base de datos scopus que corroboren experiencias ni resultados en el área. No obstante, sí se identificaron estudios relacionados con la instrucción y la capacitación en 
diferentes áreas del conocimiento, entre ellas Ingeniería y Salud, y en ocupaciones como la construcción de veleros. Una de las posibles causas de la carencia de publicaciones indexadas en bases de datos internacionales como scopus es el incipiente interés por analizar de manera empírica y reflexiva el efecto y las características de la mediación virtual en procesos de instrucción en el escenario aeronáutico.

Dentro de los principales hallazgos y aprendizajes que los estudios analizados encontraron, se resalta la caracterización de los entornos en los que se va a instruir o enseñar y de quiénes estarán expuestos a dichos aprendizajes. Esta caracterización es crucial para planear y desarrollar estrategias pedagógicas que aprovechen y optimicen de manera efectiva los EVA en favor del aprendizaje de los estudiantes. Como lo plantean Halpin et al. "la tecnología emergente está transformando no solo la forma en que los participantes aprenden, sino también la forma en que los instructores realizan un seguimiento de los datos sobre cómo los participantes estudian, usan y aprenden el contenido" (2015, p. 4). Vale la pena que futuros estudios en el tema exploren las investigaciones que han empleado guiones de enseñanza (Barbosa-Chacón \& Barbosa-Herrera, 2017), laboratorios de simulación (Singh et al., 2013) y procesos de enseñanza-aprendizaje a través de LMS 3D (Yamazaki, 2018) que optimizan los detalles de realidad virtual, pues todos ellos son pertinentes para la instrucción en el área del control de tránsito aéreo. La enseñanza-aprendizaje a través de LMS 3D, además de oportuna, es realizable si se tiene en cuenta que las instituciones del campo poseen herramientas tecnológicas (simuladores y software) que pueden usarse más extensivamente, y que a largo plazo podrían ayudar en los procesos de investigación sobre el tema. Estos procesos podrían favorecer la colaboración de investigadores nacionales con otros investigadores que desarrollen trabajos similares en otros países latinoamericanos.

Uno de los aportes más llamativos de los trabajos analizados, se refiere a la necesidad de incluir la enseñanza en gestión tecnológica o habilidades tecnológicas en los currículos de los programas virtuales. Tanto docentes como estudiantes necesitan conocer plenamente cómo funcionan los escenarios educativos virtuales en los que van a enseñar-aprender y tener experiencias situadas, relacionadas con el contenido del área disciplinar. Esto les permite apropiar mejor el conocimiento de esas tecnologías y fortalecer el uso efectivo de sus habilidades para cumplir a cabalidad con las metas de conocimiento fijadas en los programas de formación, capacitación e instrucción.

Aunque no se hallaron estudios indexados sobre la IEEPT en el área del control de tránsito aéreo, sí se identificaron rutas pedagógicas y tecnológicas que pueden ofrecer aportes significativos a estudios futuros en el campo. La instrucción virtual requiere una continua cualificación para garantizar un servicio de control de tránsito aéreo seguro. Es decir, se necesita que quienes desempeñan estas labores estén actualizados, informados, capacitados y entrenados, para lo cual la enseñanza e instrucción a través de EVA es una alternativa óptima, oportuna y valiosa.

Como aporte que sirva a las proyecciones de próximos estudios bibliométricos de esta naturaleza, valdría la pena revisar bases de datos de acceso abierto como Google Scholar, Redalyc y Scielo, ya que es posible que en el último año se hayan publicado trabajos en el área indexados en bases de datos latinoamericanas. En este estudio se emplearon los principios de un análisis bibliométrico asistido por herramientas para el procesamiento de datos bibliográficos de las publicaciones y el análisis de contenido. Los próximos trabajos pueden realizar un análisis de contenido más profundo y detallado a partir de los principios de la Teoría Fundamentada.

\section{Referencias bibliográficas}

Abutakka, R., Maciel, C., Correa, C., Martins, A., \& Spinelli, M. (2018). Innovating Citizenship and Social Control Courses through Distance Education. In Twenty-fourth Americas Conference on Information Systems, New Orleans (pp. 1-10). https://aisel.aisnet.org/amcis2018/Education/ Presentations/30/ 
Agudelo-Rivera, C., Fajardo-Acosta, N., González-Sabogal, C., Montes-Uribe, E., \& Rodríguez-Niño, N. (2019). Llegadas de turistas internacionales a Colombia durante 2001-2017: evolución, características y determinantes. Borradores de Economía, 1064, 1-42. http://repositorio. banrep.gov.co/handle/20.500.12134/9647

Amado-Salvatierra, H., Hernández, R., e Hilera, J. (2014). Teaching and Promoting Web Accessibility in Virtual Learning Environments. In 2014 IEEE Frontiers in Education Conference (FIE) Proceedings (pp. 31-34). IEEE. https:// doi.org/10.1109/FIE.2014.7044392

Anohina, A. (2005). Analysis of the Terminology Used in the Field of Virtual Learning. Journal of Educational Technology \& Society, 8(3), 91-102. https://doi.org/10.1.1.103.7742

Araiza, M. D. J., \& Leal, M. G. (2018). Directrices de educación a distancia en el siglo xxI: modalidades de aprendizaje, multimedios, diseños de instrucción y tendencias. Daena: International Journal of Good Conscience, 13(2), 132-145. http://www.spentamexico.org/v13-n2/A7.13 (2)132-145.pdf

Aria, M., Corrado, C., \& Cuccurullo, C. (2017). Bibliometrix: An R-Tool for Comprehensive Science Mapping Analysis. Journal of Informetrics, 11(4), 959-975. https://doi.org/10. 1016/j.joi.2017.08.007

Bogossian, G., Bernardino, G., \& Sousa, C. (2015). Distance Learning Courses Reviewed - What Can We See? In 7 th International Conference on Computer Supported Education CSEDU 2015 (pp. 1-10). https://doi.org/10.5220/0005491 301480155

Cherven, K. (2015). Mastering Gephi Network Visualization (1st ed.). Packt Publishing Ltd.

Clarke, A., Lewis, D., Cole, I., \& Ringrose, L. (2005). A Strategic Approach to Developing e-Learning Capability for Healthcare. Health Information and Libraries Journal, 22(Suppl 2), 33-41. https://doi.org/10.1111/j.1470-3327.2005.00611.x

Corrigan, M., Reardon, M., Shields, C., \& Redmond, H. (2008). "SURGENT"-Student e-Learning for Reality: The Application of Interactive Visual Images to Problem-Based Learning in Undergraduate Surgery. Journal of Surgical Education, 65(2), 120-125. https://doi.org/10.1016/j. jsurg.2007.11.011

Cubero, J., Ibarra, M. S., \& Rodríguez, G. (2014). A Proposal for Skill Evaluation Via Complex Tasks in Virtual Learning Environments. In TEEM '14 Proceedings of the Second International Conference on Technological Ecosystems for Enhancing Multiculturality (pp. 429-433). https://doi. org/10.1145/2669711.2669935
Daineko, Y. A., Ipalakova, M. T., Yunnikova, M. V., Brodyagina, M. A., \& Bolatov, Z. Z. (2018). Using of ICT in e-Learning: Development of the Virtual Learning Environment for Physics Study. Proceedings of Computing Conference 2017, 2018-January(July), 1195-1198. https://doi.org/10.1109/ SAI.2017.8252242

Diem, A., \& Wolter, S. C. (2013). The Use of Bibliometrics to Measure Research Performance in Education Sciences. Research in Higher Education, 54(1), 86-114. https://doi. org/10.1007/s11162-012-9264-5

Elledge, R., Houlton, S., Hackett, S., \& Evans, M. J. (2018). "Flipped Classrooms" in Training in Maxillofacial Surgery: Preparation Before the Traditional Didactic Lecture? British Journal of Oral and Maxillofacial Surgery, 56(5), 384387. https://doi.org/10.1016/j.bjoms.2018.04.006

Estévez, J. A., Castro-Martínez, J., \& Granobles, H. R. (2015). La educación virtual en Colombia: exposición de modelos de deserción. Apertura: Revista de Innovación Educativa, 7(1-9). http://www.udgvirtual.udg.mx/apertura/index. php/apertura/article/vi

Fernández, A., \& Bueno, A. (1998). Síntesis de estudios bibliométricos españoles en educación. Una dimensión evaluativa. Revista Española de Documentación Científica, 21(3), 269-285. http://dx.doi.org/10.3989/redc.1998. v21.i3.356

Flores, C., Rosecler, M., Respício, A., \& Fonseca, J. M. (2012). Training Clinical Decision-Making through Simulation. In J. E. H. et al. (eds.), Decision Support Systems - Collaborative Models and Approaches in Real Environments (pp. 59-73). Springer-Verlag Berlin Heidelberg. https://doi. org/10.1007/978-3-642-32191-7_5

García-Chitiva, M. P., \& Suárez-Guerrero, C. (2019). Estado de la investigación sobre la colaboración en Entornos Virtuales de Aprendizaje. Píxel-BIT Revista de Medios y Educación, 56, 169-191. https://doi.org/10.12795/pixelbit.2019.i56.09

Giannakou, I., Paraskeva, F., Alexiou, A., \& Bouta, H. (2016). A Case of Emotional Intelligence for Teachers' Professional Development: Emotions and Connections are Ubiquitous in Second Life. En L. Uden, D. Liberona y B. Feldmann (eds.), Learning Technology for Education in Cloud - The Changing Face of Education (pp. 39-59). Springer. https://doi.org/10.1007/978-3-319-42147-6

Gordon, N., \& Brayshaw, M. (2017). HCI International 2017 Posters' Extended Abstracts. In $\mathrm{HCl}$ International 2017 - Posters' Extended Abstracts (pp. 469-472). Springer International Publishing. https://doi.org/10.1007/978-3319-58753-0 
Green, S., Weaver, M., Voegeli, D., Fitzsimmons, D., Knowles, J., Harrison, M., \& Shephard, K. (2006). The Development and Evaluation of the Use of a Virtual Learning Environment (Blackboard 5) to Support the Learning of Pre-Qualifying Nursing Students Undertaking a Human Anatomy and Physiology Module. Nurse Education Today, 26, 388395. https://doi.org/10.1016/j.nedt.2005.11.008

Gutiérrez-Esteban, P., Yuste-Tosina, R., Arias-Masa, J., Cubo-Delgado, S., \& Alonso-Díaz, L. (2016). Evaluation of Teaching Design in Synchronous Virtual Classrooms. International Journal of Continuing Engineering Education and $\mathrm{Li}$ fe-Long Learning, 26(1), 72-89. https://doi.org/10.1504/ IJCEELL.2016.075040

Häfner, P., Vinke, C., Häfner, V., Ovtcharova, J., \& Schotte, W. (2013). The Impact of Motion in Virtual Environments on Memorization Performance. In 2013 IEEE International Conference on Computational Intelligence and Virtual Environments for Measurement Systems and Applications, CIVEMSA 2013 - Proceedings (pp. 104-109). https://doi. org/10.1109/CIVEMSA.2013.6617404

Halpin, M., Halpin, R., \& Curtis, P. (2015). Simulation-Based Electrical Safety Training. In 2015 IEEE 15th International Conference on Environment and Electrical Engineering, EEEIC 2015 - Conference Proceedings (pp. 1137-1142). Rome, Italy. https://doi.org/10.1109/EEEIC.2015.7165328

Hernández-Leo, D., Chacón, J., Prieto, L. P., Asensio-Pérez, J. I., \& Derntl, M. (2013). Towards an Integrated Learning Design Environment. En Springer (ed.), Scaling Up Learning for Sustained Impact - 8th European Conference on Technology Enhanced Learning, EC-TEL 2013, Proceedings (pp. 448-453). Springer. https://link.springer.com/ chapter/10.1007/978-3-642-40814-4_37

Jardines, F. J. (2009). Desarrollo histórico de la educación a distancia. Innovaciones de negocios, 6(2), 225-236. http://eprints.uanl.mx/id/eprint/12521

Kavitha, V., \& Lohani, R. (2018). A Critical Study on the Use of Artificial Intelligence, e-Learning Technology and Tools to Enhance the Learner's Experience. Cluster Computing, 2, 1-5. https://doi.org/10.1007/s10586-018-2017-2

Kim, E., \& Schniederjans, M. (2004). The role of in Web-based Distance Education Courses. Communications of the ACM, 47(3), 95-98. http://dx.doi.org/10.1145/971617.971622

Kleinpell, R., Wesley, E., Williams, G., Liolios, A., Ward, N., \& Tisherman, S. (2011). Web-Based Resources for Critical Care Education. Critical Care Medicine, 39(3), 541-553. https://doi.org/10.1097/CCM.0b013e318206b5b5
Larvin, M. (2009). E-Learning in Surgical Education and Training. Journal of Surgery, 8(3), 133-137. https://doi. org/10.1111/j.1445-2197.2008.04828.x

Letouze, P., Prata, D. N., Barcelos, A., Barbosa, G. V., Franca, G., \& Rocha, M. L. (2017). Is Technology Management Education a Requirement for a Virtual Learning Environment? In 2017 IEEE Technology and Engineering Management Society Conference, TEMSCON 2017 (pp. 404-408). https:// doi.org/10.1109/TEMSCON.2017.7998409

Limniou, M., Papadopoulos, N., \& Whitehead, C. (2009). Integration of Simulation Into Pre-Laboratory Chemical Course: Computer Cluster Versus Webcт. Computers \& Education, 52(1), 45-52. https://doi.org/10.1016/j.compe du.2008.06.006

López, M. V., González, C., \& Hernández, J. J. (2014). A Comparative Study of Classroom and Online Distance Modes of Official Vocational Education and Training. PLOS ONE, 9(5), 1-9. https://doi.org/10.1371/journal.pone.0096052

Lytvyn, V., Vysotska, V., Pukach, P., Bobyk, I., \& Pakholok, B. (2016). A Method for Constructing Recruitment Rules Based on the Analysis of a Specialist's Competences. Eastern-European Journal of Enterprise Technologies, 6(2), 1-14. https://doi.org/10.15587/1729-4061.2016.85454

Maxwell, S. (2012). An Agenda for uk Clinical Pharmacology: How Should Teaching of Undergraduates in Clinical Pharmacology and Therapeutics Be Delivered and Assessed? British Journal of Clinical Pharmacology, 73(6), 893899. https://doi.org/10.1111/j.1365-2125.2012.04232.x

Moazami, F., Bahrampour, E., Azar, M. R., Jahedi, F., \& Moattari, M. (2014). Comparing Two Methods of Education (Virtual Versus Traditional) on Learning of Iranian Dental Students: A Post-Test Only Design Study. BMC Medical Education, 14(45), 1-5. https://doi.org/10.1186/1472-6920-14-45

Organización de Aviación Civil Internacional (OACI). (2016). Doc 9868. Procedimientos para los servicios de navegación aérea. Instrucción. https://www.icao.int/SAM/ Documents/2016-CBT/9868_cons_es.pdf

Penafiel, M., Navarrete, R., Lujan-Mora, S., \& Zaldumbide, J. (2018). Bridging the Gaps Between Technology and Engineering Education. International Journal of Engineering Education, 34(5), 1479-1494. https://rua.ua.es/dspace/ bitstream/10045/89349/1/2018_Penafiel_etal_IJEE.pdf

Perianes-Rodriguez, A., Waltman, L., \& van Eck, N. J. (2016). Constructing Bibliometric Networks: A Comparison Between Full and Fractional Counting. Journal of Informetrics, 10(4), 1178-1195. https://doi.org/10.1016/j.joi.20 16.10.006 
Piccoli, G., Ahmad, R., \& Ives, B. (2001). Web-Based Virtual Learning Environments: A Research Framework and a Preliminary Assessment of Effectiveness in Basic IT Skills Training. MIS Quarterly, 25(4), 401-426. http://dx. doi.org/10.2307/3250989

Pinto-Llorente, A. M., Sánchez-Gómez, M. C., García-Peñalvo, F. J., \& Casillas-Martín, S. (2017). Students' Perceptions and Attitudes Towards Asynchronous Technological Tools in Blended-Learning Training to Improve Grammatical Competence in English as a Second Language. Computers in Human Behavior, 72, 632-643. https://doi. org/10.1016/j.chb.2016.05.071

RAC 65. (2019). Licencias para personal aeronáutico diferente de la tripulación de vuelo. Unidad Administrativa Especial de Aronáutica Civil. http://www.aerocivil.gov.co/normatividad/_layouts/15/WopiFrame.aspx?sourcedoc=/normatividad/RAC/RAC\%20\%2065\%20-\%20Licencias\%20 para\%20el\%20personal\%20aeron\%C3\%A1utico,\%20 \%20diferente\%20de\%20la\%20tripulaci\%C3\%B3n\%20 de\%20vuelo.pdf\&action=default

Rama, C. (2016). La fase actual de expansión de la educación en línea o virtual en América Latina. Universidades, 70, 2739. http://repositorio.minedu.gob.pe/handle/MINEDU /5114

Raupach, T., Muenscher, C., Anders, S., Steinbach, R., Pukrop, T., Hege, I., \& Tullius, M. (2009). Web-Based Collaborative Training of Clinical Reasoning: A Randomized Trial. Medical Teacher, 31(9), 431-437. https://doi.org/10.1080 01421590903095502

Raynor, M., e Iggulden, H. (2008). Online Anatomy and Physiology: Piloting the Use of an Anatomy and Physiology e-Book-VLE Hybrid in Pre-Registration and Post-Qualifying Nursing Programmes at the University of Salford. Health Information and Libraries Journal, 25(2), 98-105. https://doi.org/10.1111/j.1471-1842.2007.00748.x

San Diego, J., Cox, M., Quinn, B. F. A., Newton, J. T., Banerjee, A., \& Woolford, M. (2012). Researching Haptics in Higher Education: The Complexity of Developing Haptics Virtual Learning Systems and Evaluating Its Impact on Students'
Learning. Computers and Education, 59(1), 156-166. https://doi.org/10.1016/j.compedu.2011.11.009

Shakhovska, N., Vysotska, V., \& Chyrun, L. (2017). Intelligent Systems Design of Distance Learning Realization for Modern Youth Promotion and Involvement in Independent Scientific Researches. En N. Shakhovska (ed.), Advances in Intelligent Systems and Computing II: Selected Papers from the International Conference on Computer Science and Information Technologies (pp. 175-198). https://doi. org/10.1007/978-3-319-45991-2

Silva, M., \& López, E. B. (2015). Virtual Learning Environment in Continuing Education for Nursing in Oncology: an Experimental Study. Journal of Cancer Education, 31(4), 804810. https://doi.org/10.1007/s13187-015-0889-x

Souppez, J. B. R. G. (2015). A Student's Take on Education in the Maritime Industry. En Proceedings of the Royal Institution of Naval Architects Seminar on Education \& Professional Development (pp. 37-44). http://www.procee dings.com/48155.html

Stonebraker, P. W., \& Hazeltine, J. E. (2004). Virtual Learning Effectiveness: An Examination of the Process. The Learning Organization, 11(3), 209-225. https://doi.org/10.1 108/09696470410532987

Tovar, F. (2015). La modalidad de la educación virtual: la más adecuada para capacitar a los controladores aéreos. TecnoEsufa. Revista de Tecnología Aeronáutica, Tecnología Aeronáutica, 24, 36-41. https://publicacionesfac. com/index.php/TecnoESUFA/article/view/510

Wickham, H. (2009). ggplot2: Elegant Graphics for Data Analysis. Springer-Verlag. http://ggplot2.org

Wiecha, J., Heyden, R., Sternthal, E., \& Merialdi, M. (2010). Learning in a Virtual World: Experience With Using Second Life for Medical Education. Journal of Medical Internet Research, 12(1). https://doi.org/10.2196/jmir.1337

Yoshida, Y., Yamaguchi, S., Kawamoto, Y., Noborio, H., Murakami, S., \& Sohmura, T. (2011). Development of a Multi-Layered Virtual Tooth Model for the Haptic Dental Training System. Dental Materials Journal, 30(1), 1-6. https://doi. org/10.4012/dmj.2010-082 